\title{
Fungal cytochrome P450 monooxygenases of Fusarium oxysporum for the synthesis of $\omega$-hydroxy fatty acids in engineered Saccharomyces cerevisiae
}

Pradeepraj Durairaj ${ }^{1 \dagger}$, Sailesh Malla ${ }^{2,4 \dagger}$, Saravanan Prabhu Nadarajan ${ }^{3}$, Pyung-Gang Lee ${ }^{2}$, Eunok Jung ${ }^{2}$, Hyun Ho Park', Byung-Gee Kim² and Hyungdon Yun ${ }^{3^{*}}$

\begin{abstract}
Background: Omega hydroxy fatty acids ( $\omega$-OHFAs) are multifunctional compounds that act as the basis for the production of various industrial products with broad commercial and pharmaceutical implications. However, the terminal oxygenation of saturated or unsaturated fatty acids for the synthesis of $\omega$-OHFAs is intricate to accomplish through chemocatalysis, due to the selectivity and controlled reactivity in $\mathrm{C}-\mathrm{H}$ oxygenation reactions. Cytochrome P450, the ubiquitous enzyme is capable of catalyzing the selective terminal omega hydroxylation naturally in biological kingdom.
\end{abstract}

Results: To gain a deep insight on the biochemical role of fungal P450s towards the production of omega hydroxy fatty acids, two cytochrome P450 monooxygenases from Fusarium oxysporum (FoCYP), FoCYP539A7 and FoCYP655C2; were identified, cloned, and heterologously expressed in Saccharomyces cerevisiae. For the efficient production of $\omega$-OHFAs, the $S$. cerevisiae was engineered to disrupt the acyl-CoA oxidase enzyme and the $\beta$-oxidation pathway inactivated $(\triangle \mathrm{Pox} 1) \mathrm{S}$. cerevisiae mutant was generated. To elucidate the significance of the interaction of redox mechanism, FoCYPs were reconstituted with the heterologous and homologous reductase systems - $S$. cerevisiae CPR (SCCPR) and F. oxysporum CPR (FoCPR). To further improve the yield, the effect of pH was analyzed and the homologous FoCYP-FoCPR system efficiently hydroxylated caprylic acid, capric acid and lauric acid into their respective $\omega$-hydroxy fatty acids with $56 \%, 79 \%$ and $67 \%$ conversion. Furthermore, based on computational simulations, we identified the key residues (Asn106 of FoCYP539A7 and Arg235 of FoCYP655C2) responsible for the recognition of fatty acids and demonstrated the structural insights of the active site of FoCYPS.

Conclusion: Fungal CYP monooxygenases, FoCYP539A7 and FoCYP655C2 with its homologous redox partner, FoCPR constitutes a promising catalyst due to its high regio- and stereo-selectivity in the hydroxylation of fatty acids and in the substantial production of industrially valuable $\omega$-hydroxy fatty acids.

Keywords: Cytochrome P450, Cytochrome P450 reductase, Omega fatty acid hydroxylase, cDNA gene cloning, Heterologous expression, Saccharomyces cerevisiae

\footnotetext{
* Correspondence: hyungdon@konkuk.ac.kr

${ }^{\dagger}$ Equal contributors

${ }^{3}$ Department of Bioscience and Biotechnology, Konkuk University, Seoul,

South Korea

Full list of author information is available at the end of the article
} 


\section{Background}

Fatty acids (FA) are simple and indispensable molecules of all biological systems usually derived from triglycerides or phospholipids and exist as carboxylic acids with long unbranched saturated / unsaturated aliphatic chain molecules. The FAs are modified to generate hydroxy-, epoxy-, amino-, nitro-, and halogen- derivatives, which are building blocks for various complex molecules [1]. The hydroxylation of hydrocarbon occurring closer to the carboxyl group results in $\alpha$ - or $\beta$-hydroxylation, and in the terminal ending give rise to $\omega$-hydroxylation. Terminally oxidized omega hydroxy fatty acids ( $\omega$-OHFAs) are multifunctional compounds employed in the production of various industrial products with broad commercial and pharmaceutical implications including adhesives, lubricants, cosmetic intermediates and potential anticancer agents [2,3]. $\omega$-OHFAs derived from the medium or long chain fatty acids serve as building blocks for the synthesis of poly ( $\omega$-hydroxy fatty acids) and polymers like bioplastics with high water resistance, durability and chemical versatility $[4,5]$, which demands the substantial increase in the production of various fatty acid derivatives [1,6]. $\omega$-OHFAs are chemically procured by the cross-metathesis of unsaturated fatty acid esters, preceded by the hydroformylation and hydrogenation of the carbonyl group $[7,8]$. However, the terminal oxygenation of saturated or unsaturated fatty acids for the synthesis of $\omega$-OHFAs is intricate to accomplish through chemo catalysis, due to the selectivity and controlled reactivity in $\mathrm{C}-\mathrm{H}$ oxygenation reactions [4]. Besides, the chemical synthesis of $\omega$-OHFAs is expensive due to the formation of various byproducts that demand substantial purification strategies and affect the sustainability as it relies on severe reaction conditions and high energy demanding processes [9].

In biological systems, selective omega hydroxylation occurs naturally in mammals, plants and in certain yeast and bacteria, mostly catalyzed by the cytochrome P450 (CYP) monooxygenases [10]. Cytochrome P450, the ubiquitous enzyme forms a vast divergent family of hemethiolate proteins and performs a broad range of versatile enzymatic activities. The class II P450 enzymes along with their heme donor, cytochrome P450 reductase (CPR) execute hydroxylation of various endogenous and exogenous compounds and are involved in xenobiotic detoxification and degradation as well. Microbial cytochrome P450s are of great potential interest as they act as biocatalysts and are key elements not only for microbial natural product formation but also in bioremediation. In addition, they also play a major role as drug and agrochemical targets [11]. Cytochrome P450 enzymes are capable of catalyzing intricate reactions like regio- and stereo- selective oxidation of unactivated hydrocarbon $\mathrm{C}-\mathrm{H}$ bonds to the corresponding hydroxy $(\mathrm{C}-\mathrm{OH})$ products [12]. These $\mathrm{P} 450$ enzymes are also accountable for the initial and rate limitings step of $\mathrm{n}$ alkane and fatty acid hydroxylation [13]. Currently, the biosynthetic $\omega$-OHFAs are produced by the members of microbial CYPs like CYP52 (P450Alk) and CYP153 through the selective terminal oxygenation of fatty acids. Multiple CYP52 genes have been identified in the yeast Candida species and they encode isozymes with different or overlapping substrate specificities [13]. Nevertheless immense progress has been accomplished, low space-time yields and biocatalyst recycling affects the industrialization of these processes, which ultimately paves the way for new biotechnological production strategies.

Although various omega hydroxylase P450 monooxygenases have been identified, there are no standard reports for omega hydroxylation in the filamentous fungal kingdom. Fungal genome sequencing projects have revealed the existence of more than 6000 fungal genes coding for putative P450s which are yet to be explored for the discovery of novel catalytic enzymes $[13,14]$. These fungal CYP enzymes indulge in the biosynthesis of a vast array of secondary metabolites of biomedical, agricultural, and industrial significance [15]. With the goal of developing an alternative fungal based process to produce beneficial $\omega$-OHFAs, we investigated the novel CYPs from Fusarium oxysporum f.sp lycopersici (Fol), which is a well characterized; genome sequenced phytopathogenic fungi. In recent years, Fol also emerged as a mammalian pathogen by affecting immuno-compromised humans and mammals, and thus evolved as a dual plantmammal infection system [16]. Among the genome sequenced Fusarium strains, F. oxysporum has the largest genome size $(60 \mathrm{MB})$ comprising the greater number of protein-encoding genes $(17,735)$ as compared to its most closely related species, Fusarium graminearum $(13,332)$ and Fusarium verticillioides $(14,179)[16]$. Besides, F. oxysporum encompasses the unique bifunctional cytochrome P450s, CYP55A1 (P450nor) and CYP505A1 (P450foxy) $[17,18]$. Both P450nor and P450foxy are self-sufficient P450s; P450nor is very essential for fungal denitrification and P450foxy accounts for the $\omega-1$ to $\omega-3$ hydroxylation of fatty acids. F. oxysporum thus stands unique and signifies the molecular evolutionary path of cytochrome P450 by possessing eukaryotic CYPs with functional properties similar to those of prokaryotes.

To gain a deep insight into the biochemical role of fungal P450s in the production of omega hydroxy fatty acids, we selected two cytochrome P450 monooxygenases from $F$. oxysporum (FoCYP), FoCYP539A7 and FoCYP655C2, and heterologously expressed them in Saccharomyces cerevisiae. For the efficient production of $\omega$-OHFAs, the $S$. cerevisiae was engineered to disrupt 
the acyl-CoA oxidase enzyme and the $\beta$-oxidation pathway inactivated $(\triangle \mathrm{Pox} 1) S$. cerevisiae mutant was generated. The FoCYPs were reconstituted with the heterologous and homologous reductases $-S$. cerevisiae CPR (ScCPR), Candida albicans CPR $(\mathrm{CaCPR})$ and $F$. oxysporum $\mathrm{CPR}$ $(F o C P R)$ to elucidate the significance of the redox mechanism. Comparative analysis of the differential redox partners with the FoCYPs revealed the enhanced production and broader substrate specificity of FoCYP539A7 with FoCPR. Withal, molecular modeling studies were performed to demonstrate the structural insights of the active site of FoCYPs. To the best of our knowledge, this is the first report demonstrating the comparative analysis of heterologous and homologous reductases with the fungal omega hydroxylase cytochrome P450 monooxygenases in the synthesis of $\omega$-OHFAs (Figure 1).

\section{Results and discussion}

Gene selection and sequence analysis of FoCYP539A7 and FoCYP655C2

Fusarium oxysporum stands distinct and intrigued the noteworthy attraction for functional characterization by not only encompassing the bifunctional CYPs, P450nor and P450foxy, but also due to the inclusion of larger pool of other cytochrome P450 genes. The insilico analysis of Fusarium oxysporum f.sp lycopersici genome based on the Fungal Cytochrome P450 Database [19] revealed the presence of 169 putative cytochrome P450s suggesting that $\mathrm{Fol}$ has unique metabolic processes that are predominantly involved in both primary and secondary metabolism. To identify the $\omega$-fatty acid hydroxylase monooxygenases among the 169 putative CYPs of $F$. oxysporum (FoCYP), phylogenetic analysis was carried out with the reported $\omega$-selective or $\omega$-specific fatty acid hydroxylases (CYP52) of Candida species [12]. The phylogenetic tree generated by the neighbor-joining method showed the presence of 6 putative FoCYPs within the same gene cluster of reported CYP52 family, signifying the likelihood of sharing the conserved P450 motifs such as distal helices and substrate recognition sites towards $\omega$-FA hydroxylation (Additional file 1: Figure S1). We aimed to functionally characterize all $6 \mathrm{pu}-$ tative FoCYPs, but only the FOXG_00101, FOXG_14594 and FOXG_03506 gene candidates were amplified from the cDNA generated from the RNA cocktail mixture obtained from different day cultures of $F$. oxysporum. The FOXG_14589, FOXG_10811 and FOXG_03951 candidates were not amplified in both enriched (PDA) and minimal (nitrogen limited) medium even after repeated attempts, probably due to the lack of mRNA expression. Genomic sequence analysis revealed that FOXG_03506 gene candidate is not a full length $\mathrm{P} 450$; hence the FOXG_00101 and FOXG_14594 were subjected to functional characterization. According to Nelson's classification system, although the P450s act on the fatty acid substrates, they are classified into different CYP families based on their amino acid identity [20]. Dr.Nelson's Cytochrome P450 Database [20] has classified and designated the FOXG_00101 and FOXG_14594 candidates into the P450 superfamily as CYP539A7 and CYP655C2, respectively; and hence they are represented as FoCYP539A7 and FoCYP655C2 in this manuscript. Multiple sequence alignment analysis of FoCYP539A7 and FoCYP655C2 with the CYP52 candidates revealed the sequence similarities and showed the typical heme binding domain FNAGPRICIG and FGGGPRRCPA; respectively, in the $\mathrm{C}$ terminal region (Additional file 1: Figure S2). The sequence identity of FoCYP539A7 was found to be $42 \%$ towards CYP52A9 [21], CYP52A13 [22], CYP52A17 and CYP52A21 [23], 41\% towards CYP52A3 [21] and CYP52A4 [21], and 40\% towards CYP52A5 [21]. Correspondingly, the sequence identity of FoCYP655C2 was found to be $32 \%$ towards CYP52A9 and CYP52A21, 31\% towards CYP52A13, CYP52A17 and CYP52A3 and 30\% towards CYP52A4 and

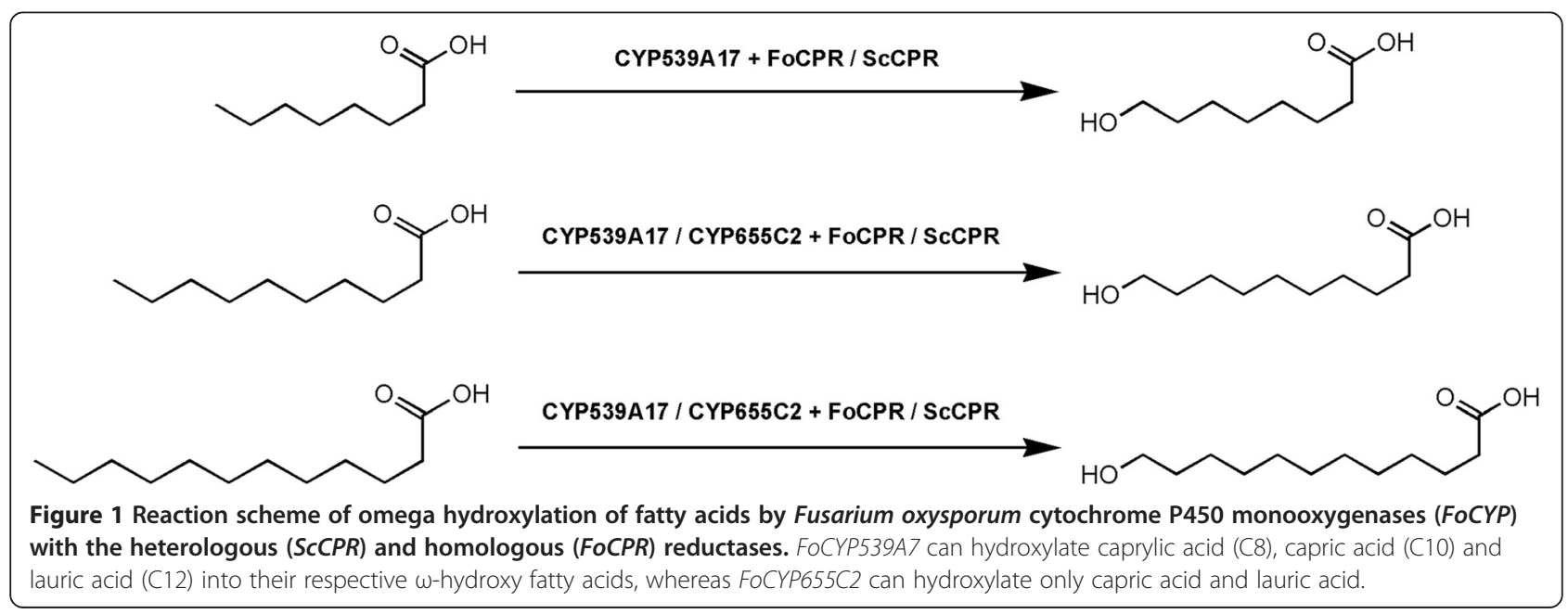


CYP52A5. The homologous nature of the FoCYP539A7 and $F o C Y P 655 C 2$ with the CYP52 family suggests the likeliness of structural and enzymatic functions towards $\omega$-FA hydroxylation.

\section{Heterologous expression and functional characterization of FoCYPs in S. cerevisiae}

For the heterologous expression of eukaryotic CYPs and for the extensive enzyme production and synthesis of value added chemicals, yeast system is the preferred host because of the presence of an endoplasmic reticulum membrane environment and the combination of higher eukaryotic protein machinery [24-28]. Hence, we aimed to heterologously express full-length FoCYP539A7 and FoCYP655C2 genes encoding 533 and 512 amino acid residues directly in the yeast S. cerevisiae BY4742 cells. The amplified FoCYP genes were cloned into pESCURA vectors and designated as pU-FoCYP539A7 and pU-FoCYP655C2 in this manuscript (Additional file 1: Figure 1A). The pU-FoCYP539A7 and pU-FoCYP655C2 vector constructs were transformed into the $S$. cerevisiae cells individually and to elucidate its heterologous expression, microsomes were isolated and $\mathrm{CO}$ difference spectral analysis was carried out. The reduced COdifference spectral analysis carried out with the yeast microsomes of FoCYP539A7 and FoCYP655C2 resulted in an absorption maximum at $448 \mathrm{~nm}$ confirming the active P450 nature (Figure 2). Based on the CO-difference spectra, the concentration of the isolated microsomes of FoCYP539A7 and FoCYP655C2 were estimated to be $0.189 \mathrm{nmol} / \mathrm{mL}$ and $0.176 \mathrm{nmol} / \mathrm{mL}$ respectively, and the active P450 obtained from a $500 \mathrm{~mL}$ yeast culture were $0.378 \mathrm{nmol}$ and $0.352 \mathrm{nmol}$ respectively. CObinding analysis performed with the microsomes of the S. cerevisiae cells harboring only the pESC-URA plasmid without FoCYP did not show any peak around $450 \mathrm{~nm}$, which confirmed the successful expression of active FoCYP539A7 and FoCYP655C2, and also demonstrated the lack of interference of intrinsic yeast CYPs due to their low levels of expression.

The sole functional activity of CYPs depends mainly on their accessory protein partner CPR for the electron transfer from NADPH to the heme group of CYPs. The $\mathrm{NADPH}$ reductase from yeast is a highly efficient and prominent redox donor for transferring electrons to various heterologous CYPs. To compare the interference of CPR over the catalytic efficiency of FoCYPs, the well reported yeast NADPH reductases from $S$. cerevisiae $(S c C P R)$ [29] and C. albicans (CaCPR) [30] were employed. The $S c C P R$ and $C a C P R$ reductase genes encoding 691 and 680 amino acid residues amplified from the respective genomic DNA were cloned into the pESC-LEU vector and designated as pL-ScCPR and pL-CaCPR, respectively (Additional file 1: Figure 1B). The CPR vector constructs was transformed and reconstituted individually into the yeast $S$. cerevisiae cells harboring pU-FoCYP539A7 and $\mathrm{pU}-\mathrm{FoCYP} 655 \mathrm{C} 2$ for co-expression and functional analysis. The yeast reconstituted system harboring $\mathrm{pU}$ FoCYP539A7 and $\mathrm{pL}-\mathrm{ScCPR} / \mathrm{pL}-\mathrm{CaCPR}$ gene constructs were termed CYP539A7-ScCPR and CYP539A7-CaCPR respectively in this manuscript. Similarly, the yeast reconstituted systems harboring the pU-FoCYP655C2 and $\mathrm{pL}-\mathrm{ScCPR} / \mathrm{pL}-\mathrm{CaCPR}$ gene constructs were termed CYP655C2-ScCPR and CYP655C2-CaCPR respectively.

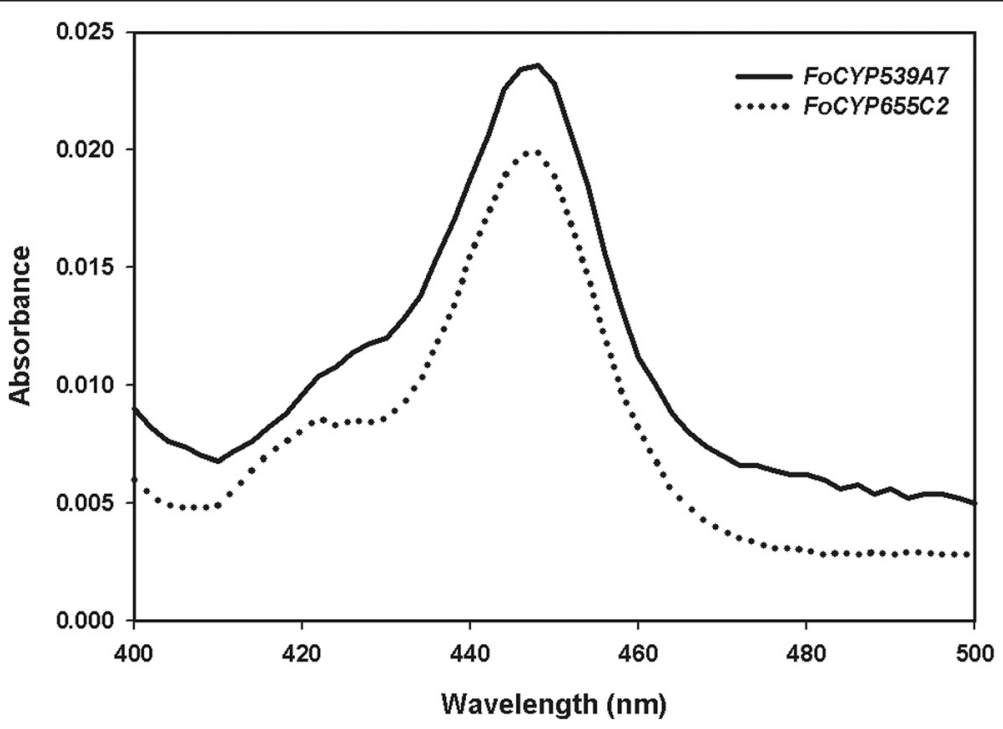

Figure 2 CO Binding analysis of microsomes of FoCYP539A7 and FoCYP655C2 expressed in S. cerevisiae. The solid line represents FOCYP539A7 and the dotted line represents FoCYP655C2. Yeast expression was carried out in S. cerevisiae cells using $4 \%$ galactose, 2 mM 5-ALA at $30^{\circ} \mathrm{C}$. 
Initially, the substrate specificity and functional catalytic efficiency of FoCYP539A7 and FoCYP655C2 reconstituted systems were analyzed both in an in vitro system and in a resting cell system with the medium and long chain fatty acids: lauric acid (C12), myristic acid (C14) and palmitic acid (C16) using $100 \mu \mathrm{M}$ substrate concentration. Microsomes were isolated from all the reconstituted systems of $S$. cerevisiae cells and the in vitro reactions were performed with the standard assay mixture. Upon incubation, the products were extracted and derivatized with BSTFA for gas chromatographic analysis. However, we were not able to observe any quantifiable data in GC analysis, probably due to the instability of the microsomal proteins and the low expression levels of the fungal cytochrome P450 systems. Subsequently, the resting cell reaction was carried out with galactose induced reconstituted systems (as mentioned above) of $S$. cerevisiae cells $(\sim 400 \mathrm{mg} / \mathrm{mL})$ in both Tris- $\mathrm{HCl}$ and potassium phosphate buffer $(\mathrm{pH} 7.0)$ with $2 \%$ dextrose or galactose. Nevertheless, GC analysis of the trimethylsilylated reaction samples did not show any significant substrate consumption or product formation in any of the reconstituted systems. This could be possibly due to the fact that the P450 being an unstable enzyme, it might have degraded during the enzyme reaction or perhaps the NADPH required for the monooxygenase reaction was not sufficient enough to produce any catalytic conversion.

To overcome this, the growing whole cell (biotransformation) system was employed, since the growing cells permit less stable enzymes like cytochrome P450 to be expressed sustainably [28]. Biotransformation was carried out with the $S$. cerevisiae cells harboring CYP539A7-ScCPR, CYP539A7-CaCPR, CYP655C2-ScCPR and CYP655C2-CaCPRsystems, which were induced with $4 \%$ galactose with $2 \mathrm{mM} 5$-ALA. C12, C14 and C16 fatty acids were added to the growing cells in $500 \mu \mathrm{M}$ substrate concentrations and the $\mathrm{pH}$ of the culture was continually maintained at $\mathrm{pH} 7.0$ throughout the reaction. In the biotransformation carried out with long chain fatty acids (LCFA) such as myristic acid and palmitic acid, GC analysis of the trimethylsilylated reaction samples did not show any substrate consumption or product formation in any of the reconstituted systems. Interestingly, the biotransformation reaction samples of lauric acid in the CYP539A7-ScCPR and CYP655C2-ScCPR reconstituted systems showed significant substrate consumption, suggesting the possible involvement of FoCYPs with medium chain fatty acids (MCFA). However, no substrate consumption was observed in the case of CYP539A7-CaCPR and CYP655C2-CaCPR reconstituted systems probably due to the lack of compatibility of CaCPR with the FoCYPs. Correspondingly, no significant changes were obtained in the biotransformation carried out with the $S$. cerevisiae cells harboring only $\mathrm{pU}$ FoCYP539A7 and pU-FoCYP655C2 constructs (control), signifying the lack of interference of intrinsic endogenous reductase with the fungal FoCYPs. Thus, the substrate consumption obtained in the CYP539A7-ScCPR and CYP655C2-ScCPR could be expounded as the result of catalytic reaction of $F o C Y P \mathrm{~s}$ with the $S c C P R$. To verify the stability of $\omega$-OHFAs in S. cerevisiae BY4742 cells, $\omega$ hydroxy lauric acid was fed to yeast systems harboring only pU-FoCYP539A7 and pU-FoCYP655C2 constructs (control) and cultured. The GC analysis of the $48 \mathrm{hr}$ culture samples did not show any product peak elucidating that $\omega$ OHFAs might have degraded naturally by the yeast.

\section{Construction of $\triangle \mathrm{Pox} 1$ mutant S. cerevisiae and synthesis of $\omega$-OHFAs}

It is indispensable to consider the fact that in yeast systems the exogenously supplied fatty acids could be degraded in two different oxidation pathways: $\omega$-oxidation in endoplasmic reticulum and $\beta$-oxidation in peroxisomes [26,31] (Additional file 1: Figure S4). The major constraint in yeast cell factory is that $\omega$-oxidation is an alternative pathway to the $\beta$-oxidation, which becomes prominent when the latter is defective [2,32]. In the biotransformation carried out with the CYP539A7-ScCPR and CYP655C2-ScCPR systems, the $\omega$-hydroxylated lauric acid could have degraded by the $\beta$-oxidation pathway of yeast cells, resulting in no product peak in the GC analysis. This provoked us to inactivate the $\beta$-oxidation pathway in the $S$. cerevisiae cells for better substrate availability to the heterologously expressed P450 enzymes and for the stability of hydroxylated fatty acids. The $\beta$-oxidation process is primarily comprised of four enzymes: acyl-CoA oxidase, enol-CoA hydratase, 3hydroxy acyl-CoA dehydrogenase and 3-oxoacyl-CoA thiolase. The first and rate-limiting enzyme in this pathway is acyl-CoA oxidase, which is encoded by a single copy gene pox1 in S. cerevisiae (Additional file 1: Figure S4). Sequential gene disruption of the acyl-CoA oxidase enzymes results in the functional blockage of the $\beta$ oxidation pathway thereby preventing the yeasts from utilizing fatty acids as a carbon source for cell growth. Inactivation of $\beta$-oxidation pathway thus becomes an attractive strategy in the metabolic engineering of yeast for the efficient production of $\omega$-OHFAs from renewable sources [33]. Using PCR-mediated gene disruption technique, we deleted the chromosomal pox1 from $S$. cerevisiae INVSc1 for the most efficient blockage of the $\beta$-oxidation pathway and the pox 1 disrupted mutant was named S. cerevisiae $\triangle$ Pox1 (Figure 3). Upon PCR amplification, only $1.4 \mathrm{~kb}$ sized gene band was obtained from the mutant strains, which confirmed the deletion of chromosomal pox1 gene (Additional file 1: Figure S5). Notwithstanding, the development or engineering of the expression host is a prerequisite for the significant improvement in the production yields of $\omega$-OHFA. 

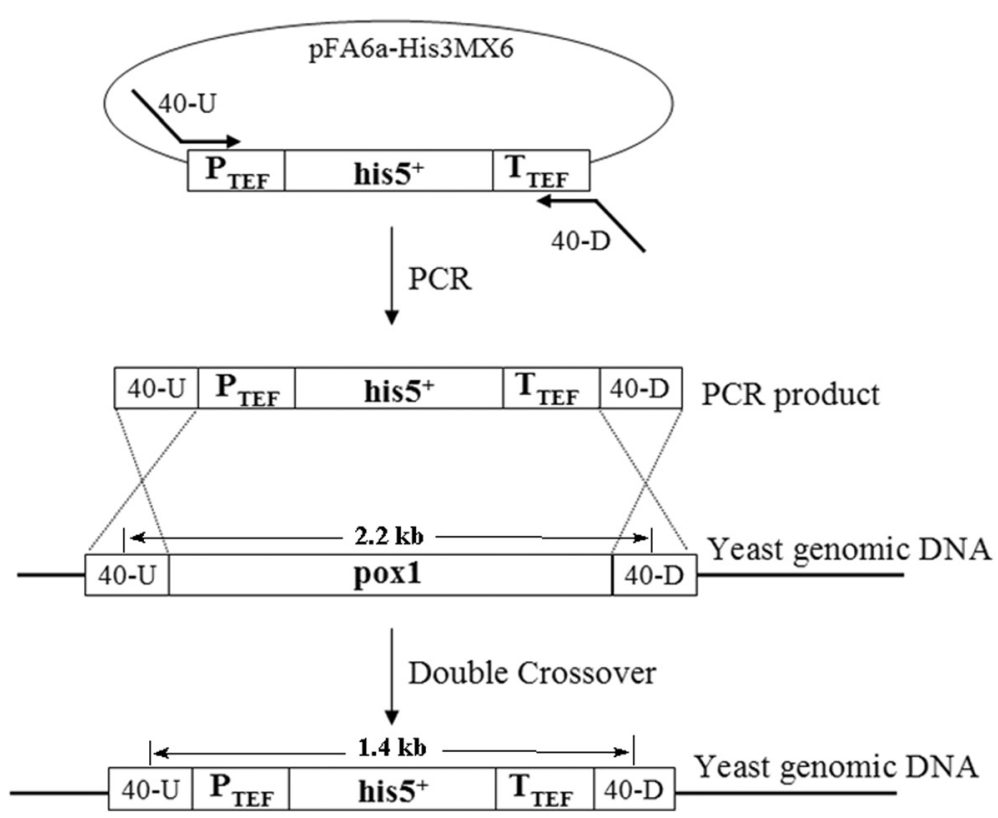

Figure 3 Schematic representation of the strategy used to disrupt pox 1 gene of S. cerevisiae INVSc1 by PCR-mediated short-region homologous recombination. The HisMX cassette was used to replace the poxigene. The double alleles of pox 1 are replaced by the HisMX auxotrophic marker through homologous recombination

The pU-FoCYP539A7 and pU-FoCYP655C2 vector constructs were retransformed and reconstituted individually into the $\Delta$ Pox1 mutant $S$. cerevisiae cells along with the pL-ScCPR for co-expression and functional analysis. GC analysis of the trimethylsilylated biotransformation samples of CYP539A7-ScCPR and CYP655C2-ScCPR reconstituted systems showed hydroxylation of lauric acid into $\omega$ hydroxy lauric acid with $42.6 \%$ and $24.9 \%$ conversion (Figure 4). The significant hydroxylation of lauric acid by the FoCYP539A7 and FoCYP655C2 enzymes stimulated us to examine the other MCFAs including caproic acid (C6), caprylic acid (C8) and capric acid (C10).

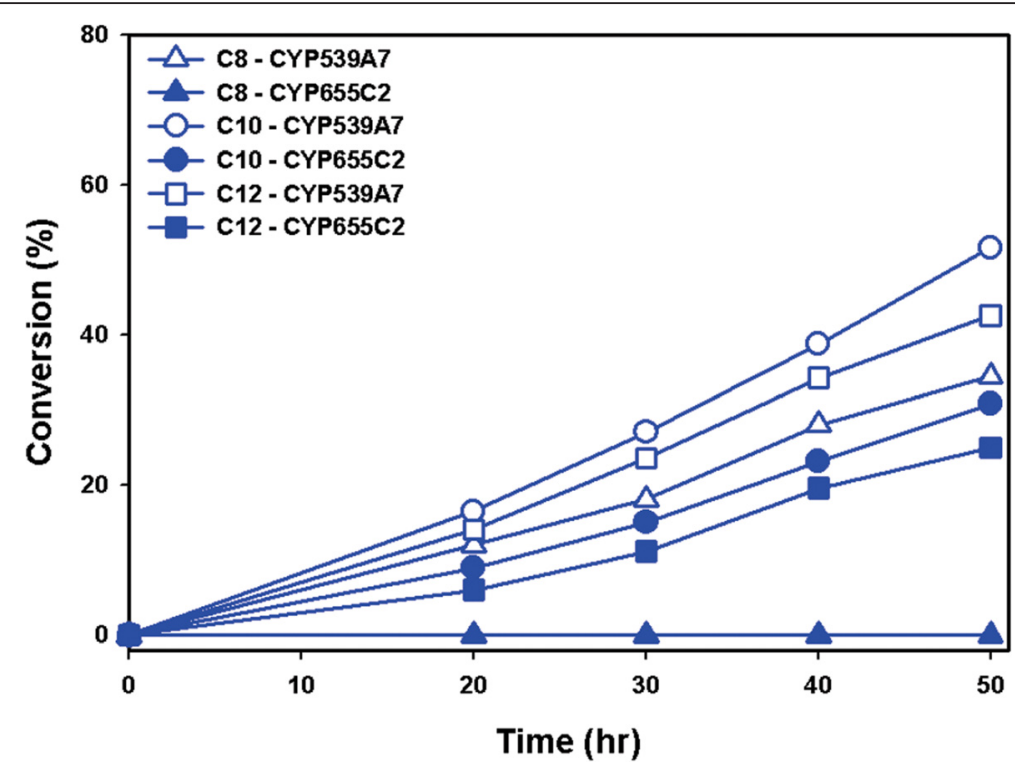

Figure 4 Reaction profiles of hydroxylation of fatty acids by FoCYP539A7 and FoCYP655C2 with the heterologous (SCCPR) reductase. $\triangle$ Pox1 mutant S. cerevisiae cells harboring the CYP39A7-SCCPR and CYP655C2-ScCPR reconstituted systems were induced with 4\% galactose, 2 mM 5-ALA and $500 \mu \mathrm{M}$ of substrates: caprylic acid (C8), capric acid (C10) and lauric acid (C12) were added and cultured at pH 7.0. Samples collected at $10 \mathrm{hr}$ intervals were extracted, trimethylsilyl derivatized and analyzed by GC. 
Interestingly, FoCYP539A7 was active to both caprylic acid and capric acid, whereas FoCYP655C2 showed activity only towards capric acid. CYP539A7-ScCPR reconstituted system hydroxylated capric acid into $\omega$-hydroxy capric acid showing better conversion than lauric acid with 51.7\% conversion (Figure 4) and hydroxylated caprylic acid into $\omega$-hydroxy caprylic acid with 34.5\% conversion (Figure 4). The CYP655C2-ScCPR reconstituted system showed only the hydroxylation of capric acid with $30.8 \%$ conversion (Figure 4 ). The eukaryotic fungal CYPs, FoCYP539A7 and FoCYP655C2 enzymes thus demonstrated their selective reactivity towards medium chain fatty acid hydroxylation (Figure 5B and Additional file 1: Table S1). The S. cerevisiae $\triangle$ Pox1 mutant harboring FoCYP reconstituted systems significantly prevented the oxidation of $\omega$-OHFAs to acetyl CoA due to the inactivation of the $\beta$-oxidation pathway.
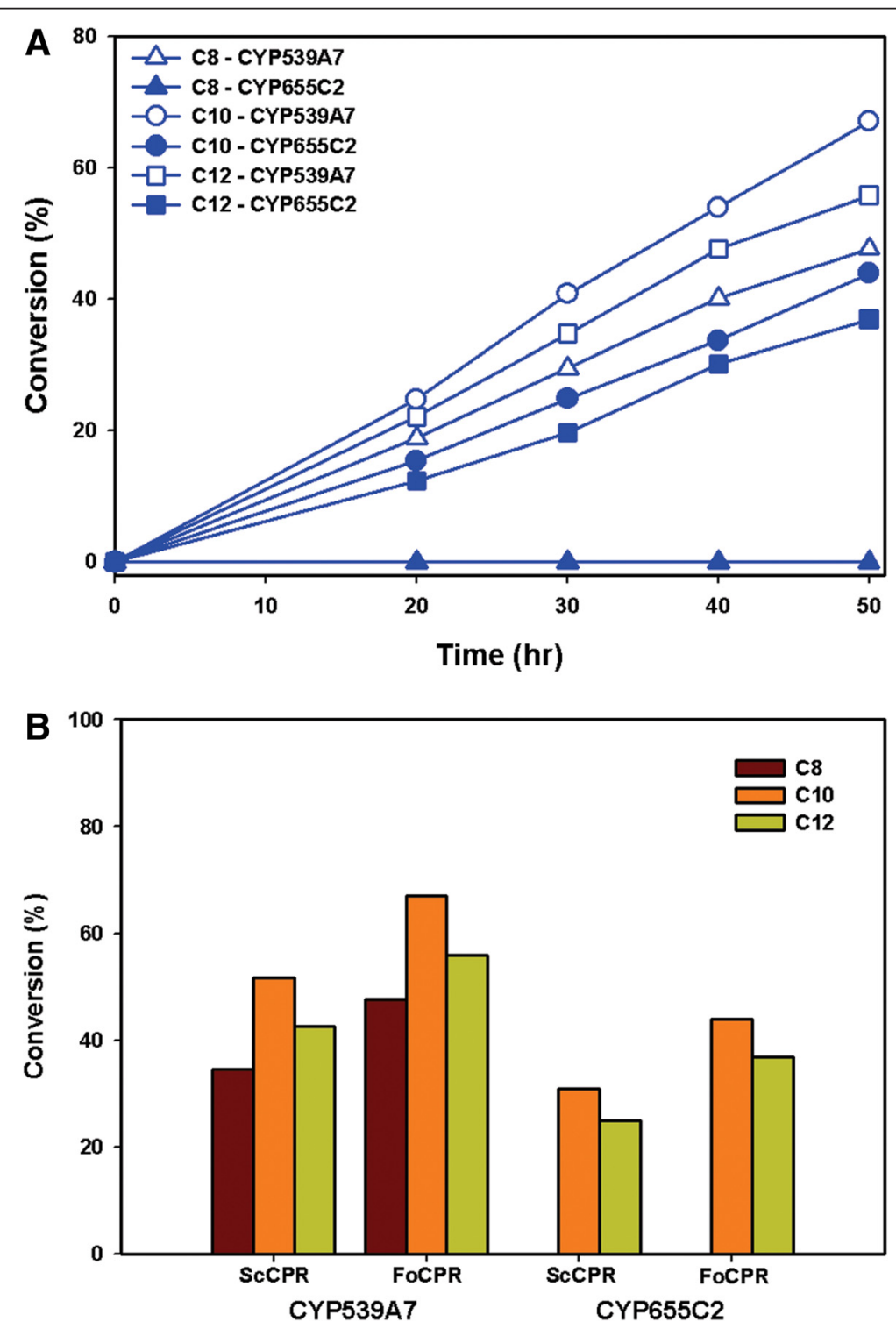

Figure 55 Significance of homologous FoCYP-FoCPR reconstituted system in the hydroxylation of fatty acids. (A) Reaction profile of hydroxylation of fatty acids by FoCYP539A7 and FoCYP655C2 with the homologous (FoCPR) reductase. (B) Comparative analysis on the catalytic conversion of fatty acids by FoCYP539A7 and FoCYP655C2 with the heterologous (ScCPR) and homologous (FoCPR) reductases. Data were plotted from the $50 \mathrm{hr}$ biotransformation reaction samples. $\triangle$ Pox 1 mutant S. cerevisiae cells harboring the CYP539A7-FoCPR, CYP655C2-FoCPR, CYP39A7-ScCPR and CYP655C2-SCCPR reconstituted systems were induced with 4\% galactose, 2 mM 5-ALA and $500 \mu M$ of substrates: caprylic acid (C8), capric acid (C10) and lauric acid (C12) were added and cultured at pH 7.0. Samples collected at $10 \mathrm{hr}$ intervals were extracted, trimethylsilyl derivatized and analyzed by GC. 
Significance of homologous FoCYP-FoCPR reconstituted system

In addition to the abundance of CYP, the sole monooxygenase reaction also relies on the abundance and electron transfer compatibility of its redox partner, CPR [34,35]. Hence, to maximize the redox coupling efficiency of P450 enzymes, co-expression with an appropriate functional CPR is crucial to achieve optimal CYP activity. For the efficient functional characterization of eukaryotic P450 genes, the homologous CYP-CPR system promotes enhanced monooxygenase activity due to their high electron transfer compatibility and coupling efficiency [34-36]. The reductase gene of $F$. oxysporum $(F o C P R)$ and its paralogues were selected from the Fusarium comparative database [16] and examined in our study. In addition to the larger number of P450 genes, filamentous fungi like $F$. oxysporum encompass multiple CPR paralogues including FOXG_08274, FOXG_03206, FOXG_ 07461 and FOXG_04834 [37]. Sequence analysis of $F$. oxysporum CPR paralogues revealed that FOXG_08274 shared a high sequence identity with the reported CPR family compared to others. We intended to employ FOXG_08274 and FOXG_07461 CPR paralogues for the functional comparative analysis, but the mRNA pertaining to FOXG_07461 was not expressed in both enriched (PDA) and minimal (nitrogen limited) medium. However, multiple sequence alignment analysis of FOXG_08247 showed the FMN-, FAD-, and NADPH- binding domains to be well conserved and homologous with the reported CPR family. Hence, the full-length FoCPR (FOXG_08247) gene encoding 692 amino acid residues amplified from the Fol cDNA was cloned into the pESC-LEU vector and designated as pL-FoCPR (Additional file 1: Figure 3B). We attempted to construct a yeast reconstituted system of FoCYP539A7 and FoCYP655C2 with its homologous CPR to compare and scrutinize its functional activity and hence the newly generated reconstituted systems were termed CYP539A7-FoCPR and CYP655C2-FoCPR respectively. Gas chromatographic analysis of the biotransformation samples of CYP539A7-FoCPR system showed significant increase in the hydroxylation of caprylic acid, capric acid and lauric acid with $47.6 \%, 67.05 \%$ and $55.8 \%$ conversion, respectively (Figure 5A and B). Similarly, the CYP655C2FoCPR system showed increased conversion of capric acid and lauric acid with $43.9 \%$ and $36.9 \%$ respectively (Figure 5A and B). The homologous FoCYP-FoCPR reconstituted system showed substantial improvement in the catalytic efficiency of both FoCYP539A7 and FoCYP655C2 enzymes (Figure 5B and Additional file 1: Table S1).

The differences in the bioconversion of fatty acid substrates between the heterologous and homologous reconstituted systems could possibly be due to the natural compatibility of FoCYPs towards the redox partner or due to the differences in the expression levels of $\mathrm{P} 450$ and CPRs [38]. Hence, parameters including the expression levels of both FoCYP539A7 and FoCYP655C2, and the redox donors ScCPR and FoCPR in all the reconstituted systems were analyzed. Microsomes were isolated from the $S$. cerevisiae cells harboring CYP539A7-ScCPR, CYP539A7-FoCPR, CYP655C2-ScCPR and CYP655C2FoCPR, and the total microsomal protein concentrations were calculated by bradford assay. Based on CO-binding analysis, the concentration of P450 in the CYP539A7ScCPR and CYP539A7-FoCPR reconstituted systems were $0.115 \mathrm{nmol} / \mathrm{mL}$ and $0.137 \mathrm{nmol} / \mathrm{mL}$ respectively (Additional file 1: Figure S6A), while the CYP655C2ScCPR and CYP655C2-FoCPR reconstituted systems had P450 concentrations of $0.081 \mathrm{nmol} / \mathrm{mL}$ and $0.112 \mathrm{nmol} /$ $\mathrm{mL}$ respectively (Additional file 1: Figure $\mathrm{S} 6 \mathrm{~B}$ ). Due to the possibility of loss of some fraction of $\mathrm{P} 450$ during the isolation procedure, the amount of $\mathrm{P} 450$ in the isolated microsomes was normalized based on the total microsomal protein concentration. The specific amounts of $\mathrm{P} 450$ in the microsomes containing CYP539A7-ScCPR, CYP539A7FoCPR, CYP655C2-ScCPR and CYP655C2-FoCPR reconstituted systems were estimated to be $1.8,1.85,1.4$ and $1.6 \mu \mathrm{mol}$ of $\mathrm{P} 450 / \mathrm{mg}$ of total protein respectively, demonstrating that the expression level of P450s in all the reconstituted systems was similar. Further, to compare the expression level of CPRs, we carried out the MTT reduction assay, where MTT (tetrazolium salt) was used as a substrate to measure the reduction activity of all coexpressed CPRs [39,40]. Equal amounts of total microsomal protein $(10 \mu \mathrm{g} / \mathrm{mL})$ of each reconstituted system were treated with MTT and the color change was observed following the addition of NADPH (Additional file 1: Figure S6C). Microsomes containing only FoCYP539A7 and FoCYP655C2 did not show any color change due to their inability to reduce MTT in the absence of CPR. The reduction of MTT into blue formazon was measured at $610 \mathrm{~nm}$ and an extinction coefficient of $11.3 \mathrm{mM}^{-1} \mathrm{~cm}^{-1}$ was used to calculate the number of moles of MTT reduced. The rate of reduction of MTT by microsomes containing CYP539A7-ScCPR, CYP539A7-FoCPR, CYP655C2-ScCPR and CYP655C2-FoCPR were $10.01 \mu \mathrm{M} / \mathrm{min}, 10.2 \mu \mathrm{M} / \mathrm{min}$, $9.9 \mu \mathrm{M} / \mathrm{min}$ and $9.5 \mu \mathrm{M} / \mathrm{min}$ respectively (Additional file 1 : Figure S6D). The MTT reduction rate demonstrates that the expression levels of heterologous and homologous reductases in all the reconstituted systems were in the same range. Despite the fact that the residue sites pertaining to the substrate specificity reside in the active site of the P450, interaction of the CPR also plays a role in the outcome of CYP reactions [41]. Therefore, it can be derived that the variation in the catalytic efficiency of FoCYP539A7 and FoCYP655C2 between the heterologous and homologous reconstituted systems is due to the interaction of CYP-CPR coupling efficiency and the electron transfer compatibility. 
The source of the reductase thus played a crucial role in the efficiency of the coupled reaction mediated by cytochrome $\mathrm{P} 450$ in terms of $\omega$-OHFAs production. Hence, the functional activity of FoCYPs is highly influenced and administered by its homologous redox partner, FoCPR.

\section{Influence of $\mathrm{pH}$ on bioconversion}

To determine the influence and effect of $\mathrm{pH}$ on the bioconversion process, the $\mathrm{pH}$ of the growing whole cell reactions was continually adjusted to 5.5 , the optimal $\mathrm{pH}$ for S. cerevisiae cell growth. It is noteworthy that the quantitative analysis of the biotransformation reaction carried out in pH5.5 showed a significant increase in the rate of product formation. The homologous CYP539A7FoCPR and CYP655C2-FoCPR reconstituted systems showed increased hydroxylation of capric acid with $78.5 \%$ and $55.5 \%$ conversion, lauric acid with $66.7 \%$ and $51.5 \%$ conversion and caprylic acid with $56.1 \%$ conversion (Figure 6 and Additional file 1: S7A). Similarly the heterologous CYP539A7-ScCPR and CYP655C2-ScCPR reconstituted systems also showed increased hydroxylation of capric acid with $61.4 \%$ and $40.9 \%$ conversion, lauric acid with $55.4 \%$ and $38.4 \%$ conversion and caprylic acid with $45.3 \%$ conversion (Additional file 1: Figure S7B and S8). The $\mathrm{pH}$ 5.5, being an optimal condition for $S$. cerevisiae cell growth, could possibly indulge in the enhanced production of heterologously expressed P450 enzymes thereby favoring better product formation (Additional file 1: Table S1). Besides to verify the influence of $\mathrm{pH}, \omega$-hydroxy fatty acids were fed to the $\Delta \mathrm{Pox} 1$ mutant S. cerevisiae cells harboring only FoCYP without CPR (control) in both pH 5.5 and $\mathrm{pH} 7.0$ culture conditions and the $24 \mathrm{hr}$ samples were extracted and analyzed by GC. Interestingly, the $\mathrm{pH} 5.5$ culture sample retained about $81.6 \% \omega$-OHFA, whereas $\mathrm{pH} 7.0$ culture samples retained only $72.3 \%$, probably due to the degradation or consumption of $\omega$-OHFAs. The enhanced stability of $\omega-$ OHFAs in pH 5.5 could be presumed as the fact behind the increased production of $\omega$-OHFAs by both FoCYP539A7 and $F o C Y P 655 C 2$ enzymes irrespective of the reductase systems (Figure 6, S8 and Additional file 1: Table S1). The order of conversion efficiency of fatty acids into their respective omega hydroxy fatty acids by FoCYP539A7 is $\mathrm{C} 10>\mathrm{C} 12>\mathrm{C} 8$ and FoCYP655C2 is $\mathrm{C} 10>\mathrm{C} 12$. Overall, the CYP539A7-FoCPR reconstituted system showed better $\omega$-OHFAs production compared to other reconstituted systems, signifying that FoCYP539A7 with FoCPR is the better candidate in terms of substrate specificity and product formation (Figure 6 and Additional file 1: Table S1).

In addition, the trimethylsilylated metabolites were analyzed by GC-MS to qualitatively analyze the hydroxylated product. In the biotransformation with caprylic acid as a substrate, the hydroxylated TMS derivatized product displayed a mass spectrum with prominent ions at $\mathrm{m} / \mathrm{z}$ 306, 290 (M-15, loss of $\mathrm{CH}_{3}$ ), 274 (M-31, loss of $\mathrm{CH}_{4}$ and $\left.-\mathrm{CH}_{3}\right), 199$ (M-105, loss of TMSOH$\left.-\mathrm{CH}_{3}\right), 147$ $\left[\mathrm{Me}_{2} \mathrm{Si}=\mathrm{O}^{+} \mathrm{SiMe}_{3}\right], 145\left[\mathrm{HO}^{+}=\mathrm{C}\left(-\mathrm{CH}=\mathrm{CH}_{2}\right)-\mathrm{OSiMe}_{3}\right]$, $132\left[\mathrm{CH}_{2}=\mathrm{C}(-\mathrm{OH})-\mathrm{OSiMe}_{3}\right], 129\left[\mathrm{CH}_{2}=\mathrm{CH}-\mathrm{C}(=\mathrm{O})-\mathrm{O}^{+}=\right.$ $\left.\mathrm{SiMe}_{2}\right]$ and $117\left[\mathrm{CH}_{2}=\mathrm{C}(-\mathrm{OH})-\mathrm{O}^{+}=\mathrm{SiMe}_{2}\right]$ and was identified as 8-hydroxyoctanoic acid (Additional file 1: Figure

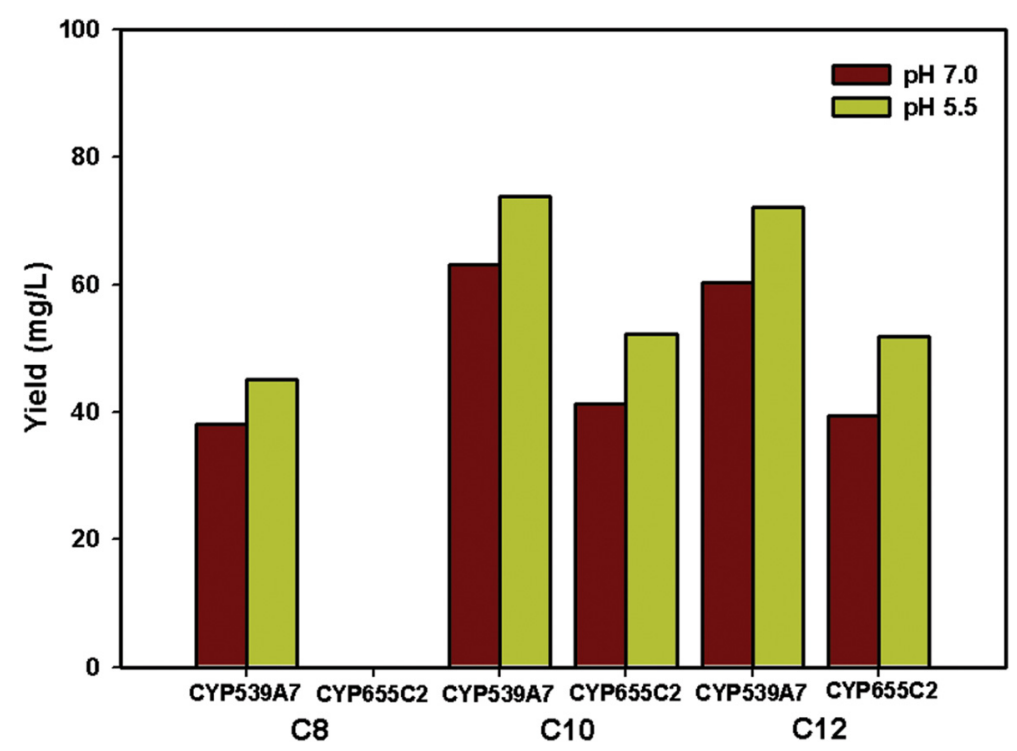

Figure 6 Final yield (mg/L) of $\omega$-hydroxy fatty acids by FoCYP539A7 and FoCYP655C2 with the homologous reductase (FoCPR) in the biotransformation carried out at $\mathrm{pH} 5.5$ and $\mathbf{p H}$ 7.0. Data were plotted from the $50 \mathrm{hr}$ biotransformation reaction samples. $\triangle \mathrm{Pox} 1 \mathrm{mutant} S$. cerevisiae cells harboring the CYP539A7-FoCPR and CYP655C2-FoCPR reconstituted systems were induced with 4\% galactose, 2 mM 5-ALA and $500 \mu \mathrm{M}$ of substrates: caprylic acid (C8), capric acid (C10) and lauric acid (C12) were added and cultured at pH 5.5 and pH 7.0. Samples collected at $10 \mathrm{hr}$ intervals were extracted, trimethylsilyl derivatized and analyzed by GC. 
S9A and S10A). With capric acid as a substrate, the hydroxylated TMS derivatized product showed a mass spectrum with prominent ions at $\mathrm{m} / \mathrm{z} 333,318$ (M-15, loss of $\left.\mathrm{CH}_{3}\right), 302\left(\mathrm{M}-31\right.$, loss of $-\mathrm{CH}_{4}$ and $\left.-\mathrm{CH}_{3}\right), 228$ (M-105, loss of TMSOH-CH $\left.\mathrm{CH}_{3}\right), 217\left[\mathrm{CH}_{2}=\mathrm{CH}-\mathrm{C}\left(=\mathrm{O}^{+}\right.\right.$ $\left.\left.\mathrm{SiMe}_{3}\right)-\mathrm{OSiMe}_{3}\right], 204\left[\mathrm{CH}_{2}-\mathrm{C}^{+}\left(-\mathrm{OSiMe}_{3}\right)-\mathrm{OSiMe}_{3}\right], 147$ $\left[\mathrm{Me}_{2} \mathrm{Si}=\mathrm{O}^{+} \mathrm{SiMe}_{3}\right], 145\left[\mathrm{HO}^{+}=\mathrm{C}\left(-\mathrm{CH}=\mathrm{CH}_{2}\right)-\mathrm{OSiMe}_{3}\right]$, $132\left[\mathrm{CH}_{2}=\mathrm{C}(-\mathrm{OH})-\mathrm{OSiMe}_{3}\right], 129\left[\mathrm{CH}_{2}=\mathrm{CH}-\mathrm{C}(=\mathrm{O})-\mathrm{O}^{+}=\right.$ $\left.\mathrm{SiMe}_{2}\right]$ and $117\left[\mathrm{CH}_{2}=\mathrm{C}(-\mathrm{OH})-\mathrm{O}^{+}=\mathrm{SiMe}_{2}\right]$ and was identified as 10-hydroxydecanoic acid (Additional file 1: Figure S9B and S10B). When lauric acid was used as a substrate, the hydroxylated TMS derivatized product showed a mass spectrum with prominent ions at $\mathrm{m} / \mathrm{z}$ 361, 346 (M-15, loss of $\left.\mathrm{CH}_{3}\right), 330$ (M-31, loss of $-\mathrm{CH}_{4}$ and $\left.-\mathrm{CH}_{3}\right), 256(\mathrm{M}-105$, loss of TMSOH$\left.-\mathrm{CH}_{3}^{-}\right), 217 \quad\left[\mathrm{CH}_{2}=\mathrm{CH}-\mathrm{C}\left(=\mathrm{O}^{+} \mathrm{SiMe}_{3}\right)-\right.$ $\left.\mathrm{OSiMe}_{3}\right], 204\left[\mathrm{CH}_{2}-\mathrm{C}^{+}\left(-\mathrm{OSiMe}_{3}\right)-\mathrm{OSiMe}_{3}\right], 147\left[\mathrm{Me}_{2} \mathrm{Si}=\right.$ $\left.\mathrm{O}^{+} \mathrm{SiMe}_{3}\right], 145\left[\mathrm{HO}^{+}=\mathrm{C}\left(-\mathrm{CH}=\mathrm{CH}_{2}\right)-\mathrm{OSiMe}_{3}\right], 132\left[\mathrm{CH}_{2}=\right.$ $\left.\mathrm{C}(-\mathrm{OH})-\mathrm{OSiMe}_{3}\right], 129\left[\mathrm{CH}_{2}=\mathrm{CH}-\mathrm{C}(=\mathrm{O})-\mathrm{O}^{+}=\mathrm{SiMe}_{2}\right]$ and $117\left[\mathrm{CH}_{2}=\mathrm{C}(-\mathrm{OH})-\mathrm{O}^{+}=\mathrm{SiMe}_{2}\right]$ and was identified as 12hydroxydodecanoic acid (Additional file 1: Figure S9C and $\mathrm{S} 10 \mathrm{C})$. The ions at $\mathrm{m} / \mathrm{z} 204$ and 217 are formed via a trimethylsilyl transfer between the ether and the ester group. The MS patterns of the reaction metabolites were found to be identical to the respective standard compounds. Thus, both FoCYP539A7 and FoCYP655C2 reconstituted systems hydroxylated fatty acids at their $\omega$-positions and produced $\omega$-OHFAs demonstrating them to be omega hydroxylase monooxygenases (Figure 1).

\section{Molecular modeling studies}

Although a large number of cytochrome P450s have been reported, the 3D structure, active site information and interaction of most of the cytochrome P450s with substrates remain unclear $[42,43]$. In this study, we predicted the model structures of FoCYP539A7 and FoCYP655C2 and their interactions with fatty acid substrates were analyzed to get the structural insight of CYP reactivity. It is reported that CYP undergoes conformational changes in the active site after substrate binding [44-46]. So, here we modeled the 3D structure of FoCYPs based on the heme domain using the best templates obtained through homology search against Protein databank. The FoCYP539A7 model structure was constructed along with the heme structure using the template of Homo sapiens CYP co-crystallized with cholesterol-3-sulfate (PDB id - 2Q9F) [44] that shares $29 \%$ sequence identity (Additional file 1: Figure S11A\&B and S12). Similarly, the FoCYP655C2 was also constructed with heme using the template of Homo sapiens (PDB id - 1TQN) [46] that shares $27 \%$ sequence identity (Additional file 1: Figure S13A\&B and S14). Initially, flexible docking was carried out with its best substrate capric acid (C10) to determine the key residues responsible for the hydrogen bond interaction of our modeled FoCYPs. From the docking study, it is clear that the Asn106 of FoCYP539A7 is the key interacting amino acid to form hydrogen bond interaction with the carboxylic acid atom of capric acid (Figure 7A). This interaction helps the precise orientation of capric acid in the active sites of FoCYP539A7 and favors the omega carbon atom to face towards the ferric atom of heme, thereby favoring omega hydroxylation. Similarly, Arg235 plays the key role in FoCYP655C2 to form hydrogen bond interaction with the carboxylic acid moiety of capric acid (Figure 7B). Based on the screening, the active site pocket of both FoCYP539A7 and FoCYP655C2 residing near $5 \AA$ of docked capric acid was comprised with hydrophobic amino acids [Additional file 1: Table S2]. Further, docking of other fatty acid substrates such as C6, C8, C12 and C14 were carried out and the docked complexes favoring the similar hydrogen bond interaction as that of capric acid were exported and analyzed. In FoCYP539A7, the docked complexes of caprylic acid (C8), capric acid (C10) and lauric acid (C12) shared the same kind of interaction and orientation (Figure 7A) and the gold scores were $31.190,31.5764$ and 32.54, respectively. Unlike FoCYP539A7, only capric acid and lauric acid shared the same kind of orientation with FoCYP655C2 (Figure 7B), and the gold scores were 48.3749 and 46.0965, respectively. Due to their shorter chain lengths, $\mathrm{C} 6$ and $\mathrm{C} 8$ fatty acids lack the normal hydrophobic interaction with the active site residues. In contrast, the $\mathrm{C} 14$ fatty acid failed to show the same kind of interaction and had a different orientation due to the presence of steric hindrance between the longer chain and the heme (Figure 7B). The docking results of FoCYP539A7 and FoCYP655C2 were well correlated with our experimental results in terms of substrate specificity and bioconversion. Based on this study, we can employ further site directed or specific mutagenesis in the active site residues of FoCYPs to extend the broad range of substrates and to increase the catalytic conversion of fatty acids.

\section{Conclusion}

The first omega fatty acid hydroxylase CYP monooxygenases from $F$. oxysporum was successfully identified, cloned, heterologously expressed in the $\beta$-oxidation pathway inactivated ( $\triangle$ Pox1) S. cerevisiae mutant. Herein, we report the comparative study on the significance of heterologous and homologous CPRs in terms of functional catalytic activity of FoCYPs. The homologous CYP539A7-FoCPR and CYP655C2-FoCPR reconstituted systems produced $73.8 \mathrm{mg} / \mathrm{L}$ and 52.2 $\mathrm{mg} / \mathrm{L}$ of 10 -hydroxydecanoic acid, $72.2 \mathrm{mg} / \mathrm{L}$ and $51.9 \mathrm{mg} /$ $\mathrm{L}$ of 12-hydroxydodecanoic acid, and $45.1 \mathrm{mg} / \mathrm{L}$ of 8hydroxyoctanoic acid. Correspondingly, the heterologous 

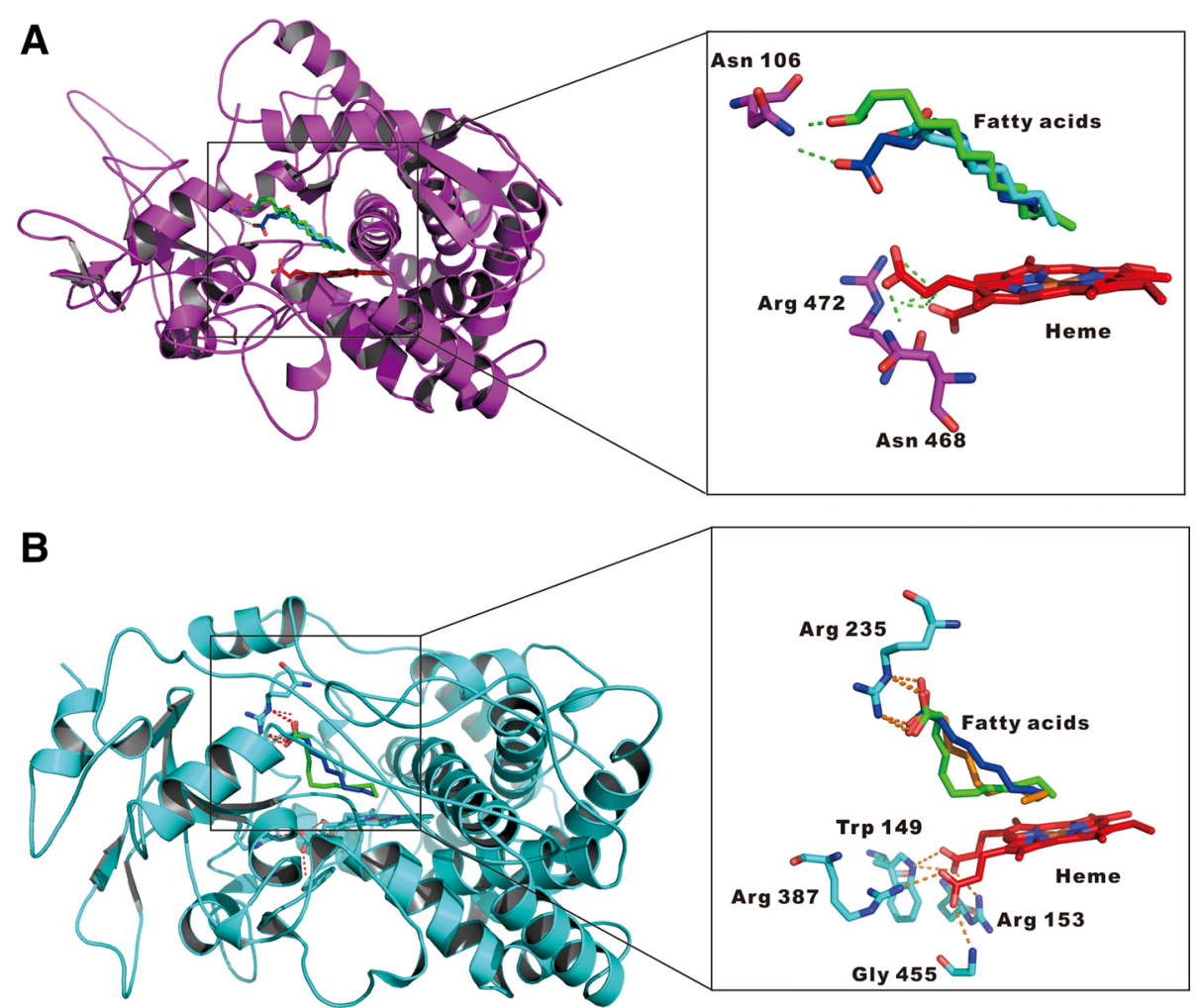

Figure 7 Superimposition of docked complexes of fatty acids in the active site of FoCYPs. (A) Superimposition of docked complexes of caprylic acid (cyan stick), capric acid (blue stick), and lauric acid (green stick) in the active site of FoCYP539A7 (pink ribbons). Fatty acids show hydrogen bond interaction with Asn106 of FoCYP539A7 and the $\omega$ carbon faces towards the ferric atom of heme. (B) Superimposition of docked complexes of capric acid (blue stick), lauric acid (green stick) and myristic acid (orange sticks) in the active site of FoCYP655C2 (cyan ribbons). Fatty acids show hydrogen bond interaction with Arg235 of FoCYP539A7 and the $\omega$ carbon faces towards the ferric atom of heme except myristic acid. The oxygen and nitrogen are represented in red and blue, and heme is represented as red sticks.

CYP539A7-ScCPR and CYP655C2-ScCPR reconstituted systems produced $57.8 \mathrm{mg} / \mathrm{L}$ and $38.5 \mathrm{mg} / \mathrm{L}$ of 10-hydroxydecanoic acid, $56.8 \mathrm{mg} / \mathrm{L}$ and $36.0 \mathrm{mg} / \mathrm{L}$ of 12-hydroxydodecanoic acid, and $36.2 \mathrm{mg} / \mathrm{L}$ of 8 hydroxyoctanoic acid. FoCYP539A7 and FoCYP655C2 with their homologous redox partner, FoCPR constitutes a promising catalyst due to its high regio- and stereo-selectivity in the substantial production of industrially valuable $\omega$-hydroxy fatty acids. In addition, we demonstrated the structural insights of active site of FoCYPs and the key residues (Asn106 of FoCYP539A7 and Arg235 of FoCYP655C2) responsible for the recognition of fatty acids based on the computational simulations. Comprehensive studies are under progress to increase the substrate specificity and production of $\omega$-OHFAs, and to elucidate the homologous redox coupling mechanism in the FoCYP mediated reactions. Subsequently, the results obtained in this study will pave the way for further biotechnological prospects to explore and exploit the novel catalytic properties of other FoCYPs.

\section{Methods}

\section{Chemicals}

All commercial chemicals including fatty acids and $\omega$ hydroxy fatty acids (C6-16), 5-aminolevulinic acid (5-ALA), amino acids were purchased from Sigma (St. Louis, MO) or Aldrich Chemical Co. (Milwaukee, WI). N,O-Bis(trimethylsilyl)-trifluoroacetamide (BSTFA) was obtained from Fluka (Buchs, Switzerland). Ethyl acetate and dimethyl sulfoxide (DMSO) were purchased from Junsei (Japan) and Duksan (Ansan, Korea) respectively. Potato dextrose (PD) media, yeast peptone dextrose (YPD) media, yeast nitrogen base w/o amino acids and luria bertani (LB) media were purchased from BD Difco (Franklin Lakes, NJ). All chemicals used were of analytical grade.

\section{Microorganism and culture conditions}

The fungal strain Fusarium oxysporum f. sp. Lycopersici strain 4287 was obtained from the Fungal Genetic Stock Centre (USA). The fungus was cultured on potato dextrose agar (PDA) for 4-5 days at $28^{\circ} \mathrm{C}$ and then cultured in potato dextrose broth (PDB) for 5-20 days under 
aerobic conditions at $150 \mathrm{rpm}$. The yeast strains used in our study are Saccharomyces cerevisiae BY4742 (YPH) (MATa his $3 \Delta 1$ leu $2 \Delta 0$ lys $2 \Delta 0$ ura $3 \Delta 0$ ) (Stratagene,

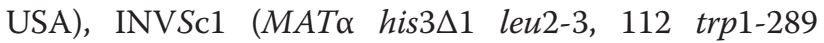
ura3-52) (Invitrogen), S. cerevisiae YSC2 (Type II, sigma) and Candida albicans SC5314. The yeast strains were grown at $30^{\circ} \mathrm{C}$ for $2-3$ days cultured in rich YPD (2\% glucose, $2 \%$ Bacto-peptone, $1 \%$ yeast extract) medium or minimal synthetic drop-out (SD) medium (2\% glucose, $0.67 \%$ yeast nitrogen base, $0.5 \%$ ammonium sulphate with all appropriate amino acids, except uracil, leucine, or both depending upon the plasmid for selection). For the induction of the galactose regulated promoters, glucose was replaced with galactose as carbon source. For the cloning and propagation of yeast plasmids, the DH5 $\alpha$ E. coli cells were cultured on the LB medium at $37^{\circ} \mathrm{C}$.

\section{Phylogenetic analysis for gene selection}

The putative cytochrome P450 gene sequences of F. oxysporum were obtained from the Fungal Cytochrome P450 Database [22]. Phylogenetic analysis was carried out with the putative FoCYPs and reported CYP52 P450s by neighbor-joining method using the Molecular Evolutionary Genetics Analysis tool (MEGA6) with the bootstrap value set to 1000. Multiple alignment was performed by using ClustalX program with the alignment parameters set to default. Sequence identity information was calculated by T-coffee software and BLAST (bl2seq) with the program set for highly similar sequences. Based on the Fusarium comparative database [16], the CPR gene of F. oxysporum (FOXG_08274) and its paralogues was selected and employed in our study.

\section{Extraction of genomic DNA, RNA and synthesis of CDNA}

Fungal mycelia were harvested from 5, 10, 15 and 20day-old cultures by vacuum filtration and frozen in liquid nitrogen. The frozen mycelia were completely ground to powder form using a mortar and pestle. The RNA was then extracted using the Qiagen RNeasy plant mini kit (Korea Ltd, Seoul) and stored at $-80^{\circ} \mathrm{C}$. The concentration of RNA was quantified at $260 \mathrm{~nm}$ using a Nanodrop (ND-1000 spectrophotometer; Thermo Fisher Scientific, DE, USA). An RNA cocktail was generated by mixing equal amounts of RNA isolated from the different days in the culture intervals. Using the RNA cocktail mixture, the first strand cDNA was synthesized with the QuantiTect reverse transcription kit, Qiagen (Hilden, Germany). The newly synthesized cDNA was stored at $-20^{\circ} \mathrm{C}$ until PCR amplification of the FoCYP and FoCPR genes. To amplify the ScCPR and CaCPR genes, genomic DNA was extracted from the $S$. cerevisiaeYSC2 and C. albicans SC5314 cells as described earlier [47].

\section{Construction of FoCYP539A7 and FoCYP655C2 reconstituted system in S. cerevisiae}

PCR amplifications were carried out using custom designed oligonucleotides (Additional file 1: Table S3 and S4) obtained from Cosmo Genetech (Seoul, Korea). The templates for the FoCYP and FoCPR genes were $F$. oxysporum cDNA, and those for ScCPR and CaCPR genes were their respective genomic DNAs. PCR was carried out using LA Taq polymerase (Takara, Japan). The annealing temperature of $54^{\circ} \mathrm{C}$ was used for the FoCYP539A7 (FOXG_00101) and FoCYP655C2 (FOXG_ 14594) genes, $61^{\circ} \mathrm{C}$ was used for $F o C P R$ and $S c C P R$ genes, and $59^{\circ} \mathrm{C}$ for the $C a C P R$ gene. The FoCYP genes were cloned into the pESC_URA vector (Stratagene, USA), and the FoCPR, ScCPR and CaCPR genes were ligated into the pESC_LEU vector (Stratagene, USA) using the SpeI and SacI restriction enzymes with the T4 DNA ligase enzyme (NEB, MA, USA). The ligated products were transformed into DH5 $\alpha$ E.coli cells and selected on LB agar medium containing $100 \mu \mathrm{g} / \mathrm{mL}$ ampicillin. The positive transformants were selected by colony PCR and restriction digestion of the cloned plasmids. The recombinant plasmids harboring the cloned genes were further confirmed by gene sequencing (Cosmo Genetech, Korea). Yeast transformations were carried out into the S. cerevisiae BY4742cells using the lithium acetate method described previously [48]. The pESC_URA plasmids harboring the FoCYP genes were transformed individually (control) and also co-transformed with the PESC_LEU plasmids harboring ScCPR, CaCPR and FoCPR. The positive transformants were selected on the minimal SD agar medium. For further confirmation of the positive transformants, plasmids were extracted from the transformed yeast cells and PCR reactions were carried out using the gene specific primers.

\section{Isolation of microsomes and $\mathrm{CO}$ difference spectral analysis}

A single yeast colony harboring FoCYP539A7 and FoCYP655C2 gene was individually inoculated into $10 \mathrm{~mL}$ of SD-U (except uracil) medium with $2 \%$ dextrose. S. cerevisiae harboring only pESC_URA plasmid without any FoCYP was used as control. The overnight grown cells were inoculated into $50 \mathrm{~mL}$ of YPG media with $4 \%$ galactose and $2 \mathrm{mM} \mathrm{5-ALA} \mathrm{to}$ obtain an $\mathrm{OD}_{600}$ of 0.4 and cultured again. The cells were collected, resuspended in $500 \mathrm{~mL}$ of fresh galactose media, and cultured for about 2 days with shaking at $150 \mathrm{rpm}$ until reaching an $\mathrm{OD}_{600}$ of 2-4. The galactose induced yeast cells were then harvested and the microsomes were isolated as described earlier [49]. UV absorbance spectra of CO-bound microsomes after sodium dithionate reduction were recorded using a UV-visible spectrophotometer (Thermo Labsystems, NY, USA) scanning between the wavelengths 400 and $500 \mathrm{~nm}$. 
Inactivation of POX1 gene in S. cerevisiae

PCR-mediated gene disruption was carried out to inactivate the acyl-CoA oxidase (pox1 gene) from S. cerevisiae INVSc1 cells. Oligonucleotides (Additional file 1: Table S3) were designed to amplify the Schizosaccharomyces pombe his $5^{+}$gene (which complements S. cerevisiae his3 mutations) with $40 \mathrm{bp}$ the flanking region on either side that had homology to the flanking region of pox1. PCR reactions were carried out using Han-pfu polymerase (Genenmed Inc., Korea) with the template DNA as plasmid pFA6a-His3MX6 [50], and the annealing temperature was set to $55-68^{\circ} \mathrm{C}$. The pFA6a plasmid containing $P_{T E F}-$ his $^{+}-T_{T E F}$ fragment was cloned using the BamHI and EcoRI restriction enzymes with the T4 DNA ligase enzyme (NEB, MA, USA). The PCR product was purified and $\sim 1.0 \mu \mathrm{g}$ of DNA was used for transformation into S. cerevisiae as described previously [50]. The selection for histidine prototrophs (transformants) was carried out on the SD medium containing adenine and the appropriate amino acids except histidine.

\section{Functional analysis of FoCYP539A7 and FoCYP655C2 reconstituted systems}

Double transformations were carried out into the $\triangle$ POX1 mutant $S$. cerevisiae to express both the FoCYP and CPR genes together. Hence, the pESC_URA plasmids harboring the FoCYP genes were co-transformed with the pESC_LEU plasmids harboring ScCPR / CaCPR / FoCPR and selected on the SD-U,-L,-H medium (except uracil, leucine and histidine). For control experiments, S. cerevisiae cells harboring only FoCYPs without any CPR were employed. Functional analysis of FoCYP539A7 and FoCYP655C2 was initially carried out in an in vitro system and a resting cell system. Later on, a biotransformation system was employed for the sustainable production of omega hydroxy fatty acids in yeast system. A single colony of yeast reconstituted system harboring both the FoCYP and CPR genes were cultured in $10 \mathrm{~mL}$ of SD-U,-L,- $\mathrm{H}$ medium with $2 \%$ dextrose and cultured at $30^{\circ} \mathrm{C}$. The overnight grown cells were then inoculated into $500 \mathrm{~mL}$ of SD or YPG media with $4 \%$ galactose and $2 \mathrm{mM} \mathrm{5-ALA}$ and cultured as described above. For the in vitro system, microsomes were isolated from all the reconstituted systems of $S$. cerevisiae cells as described earlier. The in vitro reaction was performed with the standard assay mixture containing $50 \mu \mathrm{g} / \mathrm{mL}$ of microsomal proteins, $100 \mu \mathrm{M}$ potassium phosphate buffer (pH 7.0), $500 \mu \mathrm{M}$ NADPH and $100 \mu \mathrm{M}$ of substrates (lauric acid, myristic acid and palmitic acid) and incubated at $30^{\circ} \mathrm{C}$ for 30 minutes with shaking at $150 \mathrm{rpm}$. The products were then extracted with equal volumes of ethyl acetate, dried in a vacuum concentrator and converted to their trimethylsilyl (TMS) derivatives by incubating at $50^{\circ} \mathrm{C}$ for 20 minutes with BSTFA and analyzed by gas chromatography (GC). In the resting cell system, the galactose induced cells were harvested by centrifugation $\left(3500 \mathrm{rpm}, 10 \mathrm{~min}, 4^{\circ} \mathrm{C}\right)$, washed once with $25 \mathrm{ml}$ of $100 \mathrm{mM}$ Tris- $\mathrm{HCl}$ or potassium phosphate buffer, and then resuspended in $25 \mathrm{ml}$ of $100 \mathrm{mM}$ Tris$\mathrm{HCl}$ or potassium phosphate ( $\mathrm{pH}$ 7.5) buffer. $100 \mu \mathrm{M}$ of $\mathrm{C} 12, \mathrm{C} 14$ and $\mathrm{C} 16$ substrates were added to the reaction mixture and the cells were incubated at $30^{\circ} \mathrm{C}$ for 24 hours with shaking at $150 \mathrm{rpm}$. In the biotransformation system, the overnight grown cells were then inoculated into $25 \mathrm{~mL}$ of SD or YPG media with $4 \%$ Galactose and $2 \mathrm{mM} 5$-ALA to obtain an $\mathrm{OD}_{600}$ of 0.4 and further cultured until the cells reached an $\mathrm{OD}_{600}$ of 1.0-1.2. The cells were then harvested and resuspended in fresh galactose media and $500 \mu \mathrm{M}$ of substrates: C6-C16 fatty acids were added and the cells were cultured again for 48-72 hrs. The $\mathrm{pH}$ of the growing yeast cell cultures were maintained at $\mathrm{pH} 7.0$ and $\mathrm{pH} 5.5$ for the biotransformation reactions. The reaction products were collected at different time intervals, acidified with $6 \mathrm{M}$ HCL to $c a$. pH 2 and extracted with equal volumes of ethyl acetate by vigorous vortexing and centrifugation at $14000 \mathrm{rpm}$. The reaction metabolites were then dried in the concentrator, dissolved in ethyl acetate and derivatized with BSTFA as described above. The derivatized metabolites were then analyzed by gas chromatography (GC) and mass spectrometry (MS).

\section{Product identification and quantification}

Quantitative analysis of derivatized metabolites was performed in a GC HP 6890Series (Agilent Technologies, USA) equipped with a flame ionization detector ( $\mathrm{GC} /$ FID). The sample $(2 \mu \mathrm{L})$ was injected by split mode (split ratio 20.0:1) and analyzed using a non-polar capillary column (5\% phenyl methyl siloxane capillary $30 \mathrm{~m} \times$ $320 \mu \mathrm{m}$ i.d., $0.25 \mu \mathrm{m}$ film thickness, HP-5). The oven temperature program was: $50^{\circ} \mathrm{C}$ for 1 minute, increase by $15^{\circ} \mathrm{C} / \mathrm{min}$ to $250^{\circ} \mathrm{C}$ and hold for 10 minutes. The inlet temperature was $250^{\circ} \mathrm{C}$ and for the detector, it was $280^{\circ} \mathrm{C}$. The flow rate of the carrier gas $(\mathrm{He})$ was 1 $\mathrm{mL} / \mathrm{min}$, and the flow rates of $\mathrm{H}_{2}$, air, and $\mathrm{He}$ in FID were $45 \mathrm{~mL} / \mathrm{min}, 400 \mathrm{~mL} / \mathrm{min}$, and $20 \mathrm{~mL} / \mathrm{min}$ respectively. The peaks were identified by comparison of GC chromatograms with those of authentic references.

Qualitative analysis of derivatized metabolites was performed by GC/MS using a TRACE GC ULTRA gas chromatograph (Thermo Scientific, USA), which was coupled to an ion trap mass detector ITQ1100 (Thermo Scientific, USA). The reaction sample $\left(\begin{array}{lll}1 & \mu \mathrm{L}\end{array}\right)$ was injected by splitless mode ( 0.8 minute of splitless time) and analyzed using a non-polar capillary column $(5 \%$ phenyl methyl siloxane capillary $30 \mathrm{~m} \times 250 \mu \mathrm{m}$ i.d., $0.25 \mu \mathrm{m}$ film thickness, TR-5 ms). The oven temperature program was: $50^{\circ} \mathrm{C}$ for 1 minute, increase by $15^{\circ} \mathrm{C} / \mathrm{min}$ 
to $250^{\circ} \mathrm{C}$ and hold for 10 minutes. The temperatures for inlet, mass transfer line and ion source were $250^{\circ} \mathrm{C}, 275^{\circ}$ $\mathrm{C}$, and $230^{\circ} \mathrm{C}$, respectively. The flow rate of the carrier gas $(\mathrm{He})$ was $1.0 \mathrm{~mL} / \mathrm{min}$, and the electron energy for the EI mass spectrum was $70 \mathrm{eV}$. The mass spectral peaks were identified by comparison of retention times and mass spectral data of the reaction sample with those of authentic references.

\section{Determination of expression level of CPRs by MTT assay}

The expression levels of $S c C P R$ and FoCPR were analyzed by 3-(4,5-dimethylthiazol-2-yl)-2,5-diphenyl tetrazolium bromide (MTT) assay based on its reductase activity. Microsomes were isolated from the S. cerevisiae cells harboring CYP539A7-ScCPR, CYP539A7-FoCPR, CYP655C2-ScCPR and CYP655C2-FoCPR reconstituted systems. The concentrations of the total isolated microsomal proteins were calculated based on the bradford assay and the expression level of FoCYP539A7 and FoCYP655C2 in all the reconstituted systems were subsequently estimated by $\mathrm{CO}$ binding analysis. For the MTT reductase assay, the microsomal concentrations for all the reconstituted systems were normalized to $10 \mu \mathrm{g} / \mathrm{mL}$. MTT reductase activity was carried out with $100 \mu \mathrm{M}$ MTT, $10 \mu \mathrm{g} / \mathrm{mL}$ microsomes in $100 \mathrm{mM}$ potassium phosphate buffer ( $\mathrm{pH}$ 7.6), and the reaction was initiated following the addition of $100 \mu \mathrm{M}$ NADPH [39]. The change in absorbance was measured at $610 \mathrm{~nm}$ using a UV-visible spectrophotometer (Thermo Labsystems, NY, USA) and an extinction coefficient of $11.3 \mathrm{mM}^{-1} \mathrm{~cm}^{-1}$ was used to calculate the number of moles of MTT reduced.

\section{Molecular modeling studies}

From the Fungal Cytochrome P450 Database, the translated gene sequences of FoCYP539A7 and FoCYP655C2 were retrieved and modeled using Modeler [51]. Prior to the modeling study, a protein blast search was performed against Protein structural databank (PDB) for the protein sequences FoCYP539A7 and FoCYP655C2. While modeling the protein structures, the heme from the templates were also imported using Modeler-Ligand import option. Further, the stereo chemical quality of the model was validated using SAVES server. Later, the ligand binding sites were predicted for the key residues responsible for the hydrogen bond interaction with the carbonyl oxygen of the fatty acid substrates. To identify the key residues, a flexible docking study of modeled structures with its best substrate capric acid was carried out. Correspondingly, molecular docking calculations were done for the 3D structures of the fatty acids - capric, caprylic, lauric and myristic acid with their respective modeled structure using GOLD [52]. As the fatty acid contains increased number of rotatable bonds, it can take huge number of conformation while docking in the active site. For that reason, we retrieved the different conformers of the fatty acids [caprylic (Scid-379), capric (Scid-2969), lauric (Scid-3893) and myristic acid (Scid11005)] from the Pubchem substance database. The ionization states of the fatty acids were generated using Accelrys Discovery Studio 2.1 (DS2.1; accelrysInc., CA, USA). Finally, the best docked complexes of the fatty acids showing hydrogen bond interactions with FoCYP539A7 and $F o C Y P 655 C 2$ were exported and compared for further analysis using pymol [53].

\section{Additional file}

\begin{abstract}
Additional file 1: Table S1. Final yield ( $\mathrm{mg} / \mathrm{L})$ of $\omega$-hydroxy fatty acids by FoCYPs with the heterologous (SCCPR) and homologous (FoCPR) reductases. Table S2. Active site amino acids residing $5 \AA$ of capric acid docked complexes of FoCYPs. Table S3. Oligonucleotide primers used for gene amplification and yeast expression. Table S4. Oligonucleotide primers employed in this study. Figure S1. Phylogenetic analysis of 169 putative FoCYPs with the reported $\omega$-selective or specific fatty acid CYPs. Figure S2. Multiple sequence alignment of FoCYP539A7 and FoCYP655C2 with the reported $\omega$-selective or specific fatty acid CYPs. Figure S3. Construction of yeast expression vector. Figure S4. Alternative oxidation pathways for fatty acids in yeast. Figure S5. PCR confirmation of POX1 disruption. Figure S6. Determination of expression levels of P450 and CPR in the heterologous and homologous reconstituted systems. Figure S7. Reaction profile of hydroxylation of fatty acids by FoCYP539A7 and FoCYP655C2 in the biotransformation carried out at pH 5.5. Figure S8 Final yield (mg/L) of $\omega$-hydroxy fatty acids by FoCYP539A7 and FoCYP655C2 with the heterologous reductase (SCCPR) in the biotransformation carried out at $\mathrm{pH} 5.5$ and pH 7.0. Figure S9. Representative Gas chromatographic analysis patterns of omega hydroxylated products by FoCYP539A7 and FoCYP655C2 reconstituted system. Figure S10. Representative Mass Spectral analysis patterns of omega hydroxylated products by FoCYP539A7 and FoCYP655C2 reconstituted system. Figure S11. (A) Homology modeled structure of FoCYP539A7 using 2Q9F as template. (B) Superimposed structure of model structure (cyan) and template structure-2Q9F (red). Figure S12. Ramachandran plot for modeled FoCYP539A7 derived from homology modeling. Figure S13 (A) Homology modeled structure of FoCYP655C2 using $1 \mathrm{TQN}$ as template. (B) Superimposed structure of model structure (cyan) and template structure-1TQN (red). Figure S14. Ramachandran plot for modeled FoCYP655C2 derived from homology modeling.
\end{abstract}

\section{Abbreviations}

FoCYP: Fusarium oxysporum cytochrome P450; CPR: Cytochrome P450 reductase; FA: Fatty acid; $\omega$-OHFA: Omega hydroxy fatty acid; $\triangle$ Pox1: Pox 1 deletion.

\section{Competing interests}

The authors declare that they have no competing interests.

\section{Authors' contributions}

$\mathrm{HY}$ and PD conceived and designed the study. PD performed all the experiments, analyzed the data and drafted the manuscript. SM, PGL and $E J$ assisted in the mutant strain construction. SPN assisted in the docking studies. HHP and BGK contributed on the coordination. All authors have read and approved the manuscript.

\section{Acknowledgements}

This study was supported by a grant of the Korean Health Technology R\&D Project, Ministry of Health \& Welfare, Republic of Korea (HN12C0055).

\section{Author details}

${ }^{1}$ School of Biotechnology, Yeungnam University, Gyeongsan, South Korea.

${ }^{2}$ School of Chemical and Biological Engineering, Seoul National University, 
Seoul, South Korea. ${ }^{3}$ Department of Bioscience and Biotechnology, Konkuk University, Seoul, South Korea. ${ }^{4}$ Current position: Novo Nordisk Foundation Center for Biosustainability, Technical University of Denmark, Copenhagen, Denmark.

\section{Received: 6 December 2014 Accepted: 12 March 2015} Published online: 02 April 2015

\section{References}

1. Van Bogaert IN, Groeneboer S, Saerens K, Soetaert W. The role of cytochrome P450 monooxygenases in microbial fatty acid metabolism. FEBS J. 2011;278:206-21.

2. Lu W, Ness JE, Xie W, Zhang X, Minshull J, Gross RA. Biosynthesis of monomers for plastics from renewable oils. J Am Chem Soc. 2010;132:15451-5.

3. Abe A, Sugiyama K. Growth inhibition and apoptosis induction of human melanoma cells by omega-hydroxy fatty acids. Anticancer Drugs. 2005;16:543-9.

4. Scheps D, Honda Malca S, Richter SM, Marisch K, Nestl BM, Hauer B. Synthesis of omega-hydroxy dodecanoic acid based on an engineered CYP153A fusion construct. Microb Biotechnol. 2013;6:694-707.

5. Liu C, Liu F, Cai J, Xie W, Long TE, Turner SR, et al. Polymers from fatty acids: Poly ( $\omega$-hydroxyl tetradecanoic acid) synthesis and physico-mechanical studies. Biomacromolecules. 2011;12:3291-8.

6. Kabara JJ, Swieczkowski DM, Conley AJ, Truant JP. Fatty acids and derivatives as antimicrobial agents. Antimicrob Agents Chemother 1972:2:23-8.

7. Warwel S, Jaegers H-G, Thomas S. Metathesis of unsaturated fatty acid esters - a simple approach to long-chain dicarboxylic acids. Fat Sci Technol. 1992:94:323-8

8. Metzger JO, Bornscheuer U. Lipids as renewable resources: current state of chemical and biotechnological conversion and diversification. Appl Microbiol Biotechnol. 2006;71:13-22

9. Labinger JA, Bercaw JE. Understanding and exploiting C-H bond activation Nature. 2002:417:507-14.

10. Honda Malca S, Scheps D, Kuhnel L, Venegas-Venegas E, Seifert A, Nestl BM, et al. Bacterial CYP153A monooxygenases for the synthesis of omegahydroxylated fatty acids. Chem Commun (Camb). 2012;48:5115-7.

11. Kelly SL, Kelly DE. Microbial cytochromes P450: biodiversity and biotechnology. Where do cytochromes P450 come from, what do they do and what can they do for us? Philosophical Transactions Royal Soc B: Biol Scie. 2013;368:20120476.

12. Johnston JB, Ouellet H, Podust LM, Ortiz de Montellano PR. Structural control of cytochrome P450-catalyzed omega-hydroxylation. Arch Biochem Biophys. 2011;507:86-94

13. Cresnar B, Petric S. Cytochrome P450 enzymes in the fungal kingdom. Biochim Biophys Acta. 1814;2011:29-35.

14. Park J, Park B, Jung K, Jang S, Yu K, Choi J, et al. CFGP: a web-based, comparative fungal genomics platform. Nucleic Acids Res. 2008;36:D562-71.

15. Chen W, Lee MK, Jefcoate C, Kim SC, Chen F, Yu JH. Fungal cytochrome p450 monooxygenases: their distribution, structure, functions, family expansion, and evolutionary origin. Genome Biol Evol. 2014;6:1620-34.

16. Ma LJ, van der Does HC, Borkovich KA, Coleman JJ, Daboussi MJ, Di Pietro A, et al. Comparative genomics reveals mobile pathogenicity chromosomes in Fusarium. Nature. 2010;464:367-73.

17. Nakayama N, Takemae A, Shoun H. Cytochrome P450foxy, a catalytically self-sufficient fatty acid hydroxylase of the fungus Fusarium oxysporum. J Biochem. 1996;119:435-40.

18. Nakahara K, Tanimoto T, Hatano K, Usuda K, Shoun H. Cytochrome P-450 55A1 (P-450dNIR) acts as nitric oxide reductase employing NADH as the direct electron donor. J Biol Chem. 1993;268:8350-5.

19. Park J, Lee S, Choi J, Ahn K, Park B, Park J, et al. Fungal cytochrome P450 database. BMC Genomics. 2008;9:402.

20. Nelson DR. The cytochrome p450 homepage. Hum Genomics. 2009;4:59-65.

21. Zimmer T, Ohkuma M, Ohta A, Takagi M, Schunck WH. The CYP52 multigene family of Candida maltosa encodes functionally diverse n-alkane-inducible cytochromes P450. Biochem Biophys Res Commun. 1996;224:784-9

22. Eschenfeldt WH, Zhang Y, Samaha H, Stols L, Eirich LD, Wilson $C R$, et al. Transformation of fatty acids catalyzed by cytochrome P450 monooxygenase enzymes of Candida tropicalis. Appl Environ Microbiol. 2003;69:5992-9.

23. Kim D, Cryle MJ, De Voss JJ, de Montellano PR O. Functional expression and characterization of cytochrome P450 52A21 from Candida albicans. Arch Biochem Biophys. 2007:464:213-20.

24. Pscheidt B, Glieder A. Yeast cell factories for fine chemical and API production. Microb Cell Fact. 2008;7:25.

25. Van Roermund C, Waterham H, lilst L, Wanders R. Fatty acid metabolism in Saccharomyces cerevisiae. Cellular Molecular Life Scie. 2003;60:1838-51.

26. Hiltunen JK, Mursula AM, Rottensteiner H, Wierenga RK, Kastaniotis AJ, Gurvitz A. The biochemistry of peroxisomal beta-oxidation in the yeast Saccharomyces cerevisiae. FEMS Microbiol Rev. 2003;27:35-64.

27. Huang FC, Peter A, Schwab W. Expression and characterization of CYP52 genes involved in the biosynthesis of sophorolipid and alkane metabolism from Starmerella bombicola. Appl Environ Microbiol. 2014;80:766-76.

28. Zollner A, Buchheit D, Meyer MR, Maurer HH, Peters FT, Bureik M. Production of human phase 1 and 2 metabolites by whole-cell biotransformation with recombinant microbes. Bioanalysis. 2010;2:1277-90.

29. Murakami H, Yabusaki Y, Sakaki T, Shibata M, Ohkawa H. Expression of cloned yeast NADPH-cytochrome P450 reductase gene in Saccharomyces cerevisiae. J Biochem. 1990;108:859-65.

30. Park HG, Lim YR, Eun CY, Han S, Han JS, Cho KS, et al. Candida albicans NADPH-P450 reductase: expression, purification, and characterization of recombinant protein. Biochem Biophys Res Commun. 2010;396:534-8.

31. Cherkaoui-Malki M, Surapureddi S, El-Hajj HI, Vamecq J, Andreoletti P. Hepatic steatosis and peroxisomal fatty acid beta-oxidation. Curr Drug Metab. 2012;13:1412-21.

32. Picataggio S, Rohrer T, Deanda K, Lanning D, Reynolds R, Mielenz J, et al. Metabolic engineering of Candida tropicalis for the production of long-chain dicarboxylic acids. Biotechnology (N Y). 1992;10:894-8.

33. Dmochowska A, Dignard D, Maleszka R, Thomas DY. Structure and transcriptional control of the Saccharomyces cerevisiae POX1 gene encoding acyl-coenzyme A oxidase. Gene. 1990;88:247-52.

34. Braun A, Geier M, Buhler B, Schmid A, Mauersberger S, Glieder A. Steroid biotransformations in biphasic systems with Yarrowia lipolytica expressing human liver cytochrome P450 genes. Microb Cell Fact. 2012;11:106.

35. Sono M, Roach MP, Coulter ED, Dawson JH. Heme-containing oxygenases. Chem Rev. 1996:96:2841-88.

36. Dietrich M, Grundmann L, Kurr K, Valinotto L, Saussele T, Schmid RD, et al. Recombinant production of human microsomal cytochrome P450 2D6 in the methylotrophic yeast Pichia pastoris. Chembiochem. 2005;6:2014-22.

37. Lah L, Krasevec N, Trontelj P, Komel R. High diversity and complex evolution of fungal cytochrome P450 reductase: cytochrome P450 systems. Fungal Genet Biol. 2008:45:446-58.

38. Durairaj P, Jung E, Park HH, Kim BG, Yun H. Comparative functional characterization of a novel benzoate hydroxylase cytochrome P450 of Fusarium oxysporum. Enzym Microb Technol. 2015;70:58-65.

39. Yim SK, Yun CH, Ahn T, Jung HC, Pan JG. A continuous spectrophotometric assay for NADPH-cytochrome P450 reductase activity using 3-(4,5-dimethylthiazol-2-yl)2,5-diphenyltetrazolium bromide. J Biochem Mol Biol. 2005;38:366-9.

40. Park SH, Kang JY, Kim DH, Ahn T, Yun CH. The flavin-containing reductase domain of cytochrome P450 BM3 acts as a surrogate for mammalian NADPH-P450 reductase. Biomol Ther (Seoul). 2012;20:562-8.

41. Sevrioukova IF, Poulos TL. Structural biology of redox partner interactions in P450cam monooxygenase: a fresh look at an old system. Arch Biochem Biophys. 2011;507:66-74.

42. Estrada DF, Laurence JS, Scott EE. Substrate-modulated cytochrome P450 $17 \mathrm{~A} 1$ and cytochrome b5 interactions revealed by NMR. J Biol Chem. 2013;288:17008-18.

43. Kumar S. Molecular modeling and identification of substrate binding site of orphan human cytochrome P450 4 F22. Bioinformation. 2011;7:207-10.

44. Mast N, White MA, Bjorkhem I, Johnson EF, Stout CD, Pikuleva IA. Crystal structures of substrate-bound and substrate-free cytochrome P450 46A1, the principal cholesterol hydroxylase in the brain. Proc Natl Acad Sci U S A. 2008;105:9546-51.

45. Sevrioukova IF, Poulos TL. Structural and mechanistic insights into the interaction of cytochrome P4503A4 with bromoergocryptine, a type I ligand. J Biol Chem. 2012;287:3510-7.

46. Yano JK, Wester MR, Schoch GA, Griffin KJ, Stout CD, Johnson EF. The structure of human microsomal cytochrome P450 3A4 determined by X-ray crystallography to 2.05-A resolution. J Biol Chem. 2004;279:38091-4. 
47. Harju S, Fedosyuk H, Peterson KR. Rapid isolation of yeast genomic DNA: Bust n' Grab. BMC Biotechnol. 2004:4:8.

48. Tan SL, Dossett M, Katze MG. Cryopreserved yeast cells suitable for routine, small-scale transformations. Biotechniques. 1998:25:792-4. 796.

49. Stansfield I, Kelly SL. Purification and quantification of Saccharomyces cerevisiae cytochrome P450. Methods Mol Biol. 1996:53:355-66.

50. Longtine MS, McKenzie 3rd A, Demarini DJ, Shah NG, Wach A, Brachat A, et al. Additional modules for versatile and economical PCR-based gene deletion and modification in Saccharomyces cerevisiae. Yeast. 1998;14:953-61.

51. Eswar N, Webb B, Marti-Renom MA, Madhusudhan MS, Eramian D, Shen MY, et al. Comparative protein structure modeling using MODELLER. Curr Protoc Protein Sci. 2007;2:2-9.

52. Verdonk ML, Cole JC, Hartshorn MJ, Murray CW, Taylor RD. Improved protein-ligand docking using GOLD. Proteins. 2003;52:609-23.

53. Schrödinger. The PyMOL molecular graphics system, version 1.3r1. New York: LLC; 2010.

\section{Submit your next manuscript to BioMed Central and take full advantage of:}

- Convenient online submission

- Thorough peer review

- No space constraints or color figure charges

- Immediate publication on acceptance

- Inclusion in PubMed, CAS, Scopus and Google Scholar

- Research which is freely available for redistribution 